\title{
Variant Distribution of Skin Lesions in Angiokeratoma Circumscriptum: Report of an Untypical Case
}

\author{
Huan Wang ${ }^{\mathrm{a}}$, Yan Guo Zhang ${ }^{\mathrm{a}}$, Yao Qing Yu ${ }^{\mathrm{b}, \mathrm{c}}$
}

\begin{abstract}
Angiokeratoma circumscriptum is one of rare skin lesions mainly manifested in hyperkeratotic papules or nodules occurring frequently unilaterally on the foot or leg and most characteristically takes the form of an extensive vascular plaque. Herein, we report an untypical case of angiokeratoma circumscriptum. Different from the typical form of localized extensive vascular plaque and unilaterally location of the foot or distal aspect of the leg, the skin lesions of present case initiated in the left elbow and recently progressed to ipsilateral arm and trunk. In addition, the skin lesions are varied from multiple, discrete papules to confluent, protruding verrucous plaques. The physical and histopathological findings are consistent with a diagnosis of angiokeratoma circumscriptum. The untypical distribution of skin lesions indicates that the forms of angiokeratoma circumscriptum may be varient in clinic. Since present case shares similar appearances in cutaneous vascular lesions (angiokeratomas) with systemic angiokeratoma corporis diffusum (Fabry disease), the difference between the two entities is also discussed.
\end{abstract}

Keywords: Angiokeratoma circumscriptum; Fabry disease

\section{Introduction}

Angiokeratoma circumscriptum is one of five types in the group of the angiokeratomas and clinically demonstrated in hyperkeratotic papules or nodules occurring frequently unilaterally on the leg. Recent investigations found that the

\footnotetext{
Manuscript accepted for publication May 10, 2012

${ }^{a}$ Department of Dermatology, Tangdu Hospital, Fourth Military Medical University, Xi'an 710038, P. R. China

${ }^{b}$ Institute for Biomedical Sciences of Pain, Tangdu Hospital, Fourth Military Medical University, Xi'an 710038, P. R. China ${ }^{\mathrm{c}}$ Corresponding author: Yao Qing Yu, Institute for Biomedical Sciences of Pain, Tangdu Hospital, Fourth Military Medical University, \#1 Xinsi Road, Baqiao, Xi'an, Shanxi, 710038, P. R. China. Email: yyq7803@163.com
}

doi: $10.4021 / \mathrm{jmc} 664 \mathrm{w}$ forms of skin lesions were variants and many other positions, such as buttocks [1], tongue [2], penis [3], neck [4] and even oral cavity [5] could be entangled. These lesions are usually present at birth without systemic diseases. A typical lesion becomes increasingly studded with hyperkeratotic papules, inclining to bleed on trauma, but without tendency to spontaneous improvement [6]. Furthermore, the skin lesions are often localized and the pattern of disperse distribution of is very rare.

Present untypical case showed that the skin lesions initiated in the left elbow and recently progressed to ipsilateral arm and trunk. The occurrence of disperse arrangement of skin lesions indicated the possibility of variant forms of angiokeratoma circumscriptum. Although the precise mechanism was unclear, the untypical distribution strongly supported the recently demonstrated concept that angiokeratoma circumscriptum reflects a mosaic state of a mutation [7]. The untypical distribution of skin lesions should be recognized by physicians in clinic.

\section{Case Report}

A 27-year-old female was referred to our department with complaint of variant skin lesions that had been present since birth. Initially, small red macules with occasional itching, but not pin-point bleeding, were limited to her left elbow. Gradually, her skin lesions developed into confluent, protruding verrucous plaques. After local laser treatment performed six years ago, the red-purple papules over the left anterior chest and the upper back progressed during the past two years. These papules slowly increased in number and size. There was no family history of similar lesions.

On physical examination, the patient had normal physical and mental development. Over the left elbow, there were protruding and circumscribing verrucous red-purple papules which were associated with permanent scars produced by local laser treatment. The arrangement of skin lesions was relatively localized or "segmental distribution". Clusters of individual, punctate, dark red to purple papules were found over the left upper back (Fig. 1a) and the left anterior chest (Fig. 1b). Discrete and tiny papules with no or slight hyper- 

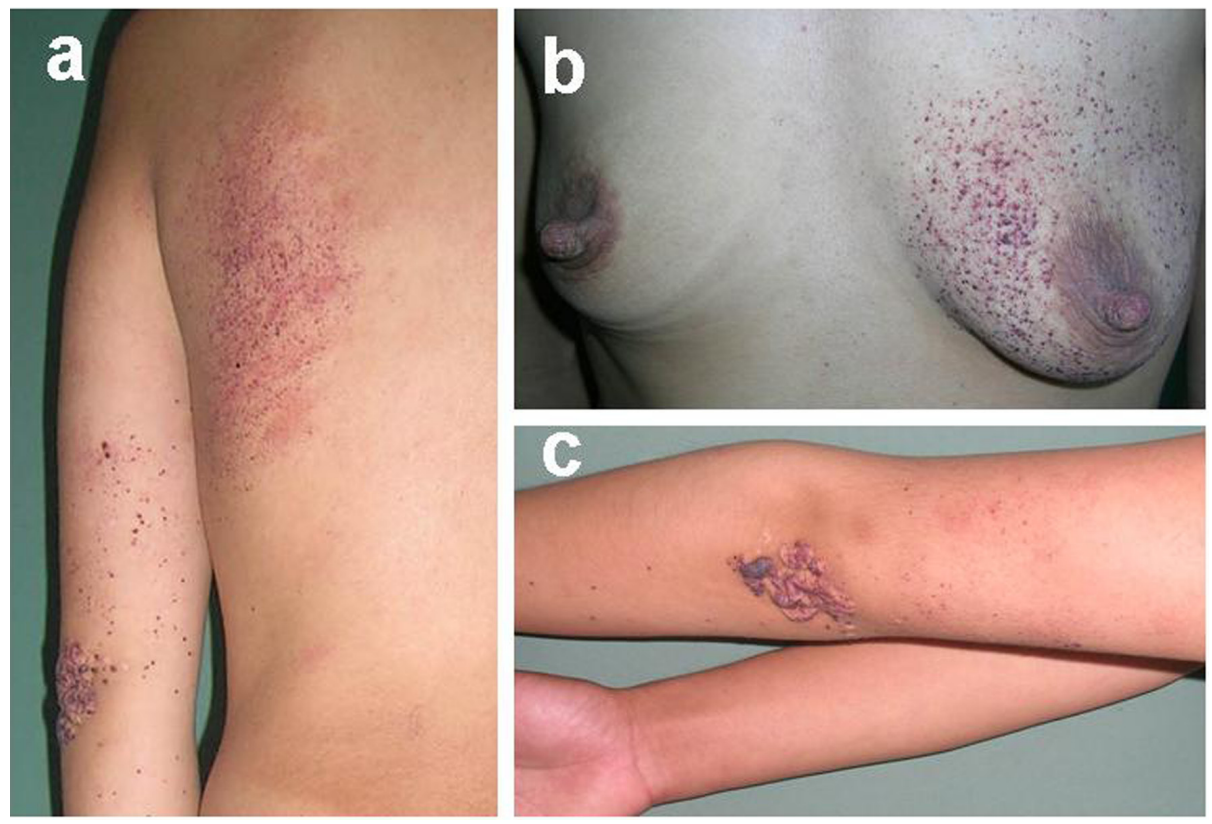

Figure 1. (a) Left upper back with clusters of individual, punctuate and dark red to purple papules. (b) Left anterior chest with multiple, tiny, red-purple and hyperkeratotic papules. (c) Localized, protruding, verrucous and red-purple papules over left elbow and discrete and tiny papules scattered along left arm. Permanent scars produced by laser treatment are also in revelation.

keratosis scattered along the left arm (Fig. 1c).

Results of light microscopy examination of a skin biopsy specimen were similar in both circumscribed and segmental lesions: hyperkeratosis of epidermis, increased thickness of the granular layer and dilation of subpapillary vessels filling with red blood cells. Lymphohistiocytic infiltrate could be found around the wall of vessels (Fig. 2).

In addition, investigations of her full blood cell count, electrolytes, renal and hepatic tests and urine sediment analysis were all within normal limits.

Based on the typical histopathological results and relatively localized position of skin lesions in the arm and trunk,

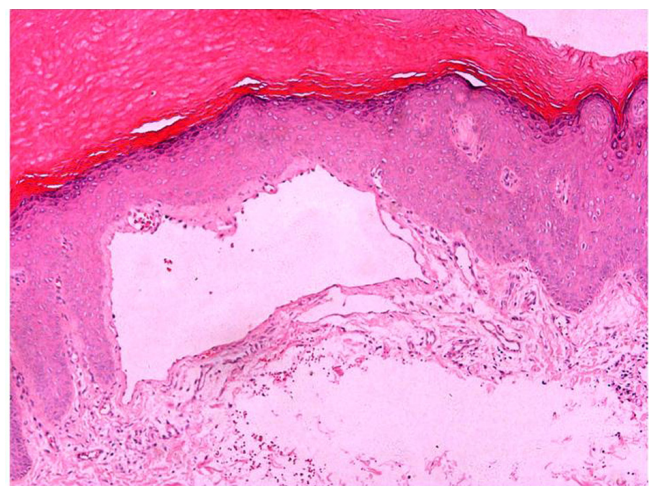

Figure 2. Microscopy (x 100) of histology of skin showed hyperkeratosis of epidermis, increased thickness of the granular layer and dilation of subpapillary vessels. Lymphohistiocytic infiltrate appeared around the wall of dilated vessel. a diagnosis of angiokeratoma circumscriptum was made.

\section{Discussion}

Angiokeratoma circumscriptum belongs to one of five types in the group of the angiokeratomas, also known as unilateral verrucous haemangioma, angiokeratoma eircumseriptum nevifome, keratotie haemangioma [8, 9]. Angiokeratoma circumscriptum is rare and most characteristically takes the form of an extensive vascular plaque, usually present from birth. The lesion is typically localized unilaterally on the foot or distal aspect of the leg. A typical lesion becomes increasingly studded with hyperkeratotic papules, and may bleed readily on trauma $[5,6]$.

Disperse distribution of angiokeratoma circumscriptum is rarely reported. We herein report a case of variant hyperkeratotic vascular lesions unilaterally arranged over the unilateral arm and ipsilateral trunk. The precise mechanisms of this untypical distribution of skin lesions are unclear, supporting the recently demonstrated concept that angiokeratoma circumscriptum reflects a mosaic state of a mutation that is so far unknown [7]. In addition, we could not exclude the possibility that incorrect medical treatment might deteriorate the disease because the skin lesions of present case progressed from left elbow to ipsilateral trunk after four years of laser treatment. Although many methods, such as diathermy or curettage and cautery for small lesions, argon laser [10], flash-lamp pumped dye laser [11], copper vapor laser [12] or surgical excision for the lager ones, are trying to cure skin 
lesions, complete healing of angiokeratoma circumscriptum has far way to go.

Recently, a case of systemic angiokeratoma corporis diffusum (Fabry disease) associated with angiokeratoma circumscriptum was reported, in which the distribution of cutaneous lesions also appeared in a diffuse pattern, with similar papules over anterior chest, back, hips, thighs, penis, and scrotum [13]. Therefore, it's necessary to clarify the difference of these two entities. Fabry disease is an inherited deficiency of $\alpha$-galactosidase-A (aGalA) enzyme due to mutations in the Gal gene at Xq22 which result in glycosphingolipids disorder [14]. Onset of the disease usually occurs during childhood or adolescence with periodic crises of severe pain in the extremities (acroparesthesias) and the appearance of cutaneous vascular lesions (angiokeratomas), hypohidrosis or anhidrosis, and characteristic corneal and lenticular opacities. The symptoms often affect male patients in adulthood. In addition to the skin lesions, stroke, heart disorders (conduction disturbances, valve disease, and left heart failure) and kidney disorders (proteinuria and chronic renal failure) often develop because of the accumulation of sphingolipids throughout the body [15]. Differently, angiokeratoma circumscriptum is a nevoid hamartomatous lesion arising early in life during infancy or childhood. Laboratory examinations on renal and hepatic tests and urine sediment analysis of present patient are all within normal limits. Therefore, the normal physical and laboratory examinations are adequate to exclude the Fabry disease from angiokeratoma circumscriptum.

Present report suggests that the forms of angiokeratoma circumscriptum may be variety in clinic and should be well recognized.

\section{Acknowledgment}

This work was supported by the National Natural Science Foundation of China $(81102064,31100803)$ and grants from Fourth Military Medical University.

\section{References}

1. del Pozo J, Fonseca E. Angiokeratoma circumscriptum naeviforme: successful treatment with carbon-dioxide laser vaporization. Dermatol Surg. 2005;31(2):232-236.

2. Varshney S. Angiokeratoma circumscriptum of the tongue. Int J Dermatol. 2005;44(10):886-888.
3. Saha M, Barlow R, Bunker CB. Angiokeratoma circumscriptum of the penis. Br J Dermatol. 2006;154(4):775776.

4. Sardana K, Koranne RV, Sharma RC, Mahajan S. Angiokeratoma circumscriptum naeviforme: rare presentation on the neck. Australas J Dermatol. 2001;42(4):294295.

5. Kumar MV, Thappa DM, Shanmugam S, Ratnakar C. Angiokeratoma circumscriptum of the oral cavity. Acta Derm Venereol. 1998;78(6):472.

6. Black MM, Grawkrodger DJ, Seymour CA, Weismann $\mathrm{K}$. Metabolic and nutritional disorders. In: Champion RH, Burton JL, Burns DA, Breathnach SM, eds. Textbook of Dermatology. Blackwell -Science, Oxford 1998; 2577-2677..

7. Bechara FG, Happle R, Altmeyer P, Grabbe S, Jansen T. Angiokeratoma circumscriptum arranged in a systematized band-like pattern suggesting mosaicism. J Dermatol. 2006;33(7):489-491.

8. Schiller PI, Itin PH. Angiokeratomas: an update. Dermatology. 1996;193(4):275-282.

9. Yang CH, Ohara K. Successful surgical treatment of verrucous hemangioma: a combined approach. Dermatol Surg. 2002;28(10):913-919; discussion 920.

10. Occella C, Bleidl D, Rampini P, Schiazza L, Rampini E. Argon laser treatment of cutaneous multiple angiokeratomas. Dermatol Surg. 1995;21(2):170-172.

11. Grekin RC, Flynn TC, Cooper D, Geisse J. Efficacy of a 2-mm spot size lens for the treatment of superficial vascular lesions with a flashlamp-pumped dye laser. Int J Dermatol. 1997;36(11):865-869.

12. Lapins J, Emtestam L, Marcusson JA. Angiokeratomas in Fabry's disease and Fordyce's disease: successful treatment with copper vapour laser. Acta Derm Venereol. 1993;73(2):133-135.

13. Sodaifi M, Aghaei S, Monabati A. Cutaneous variant of angiokeratoma corporis diffusum associated with angiokeratoma circumscriptum. Dermatol Online J. 2004;10(1):20.

14. Desnick RJ, Brady R, Barranger J, Collins AJ, Germain DP, Goldman M, Grabowski G, et al. Fabry disease, an under-recognized multisystemic disorder: expert recommendations for diagnosis, management, and enzyme replacement therapy. Ann Intern Med. 2003;138(4):338346.

15. Masson C, Cisse I, Simon V, Insalaco P, Audran M. Fabry disease: a review. Joint Bone Spine. 2004;71(5):381383. 